Original paper

\title{
Sepsis outcomes in patients with pre-existing liver disease
}

\author{
Maja Kopczynska1,2, Tamas Szakmany ${ }^{1,3}$ \\ 'Department of Anaesthesia, Intensive Care and Pain Medicine, Division of Population Medicine, Cardiff University, Heath Park Campus, Cardiff, UK \\ 2Salford Royal NHS Trust, Stott Lane, Manchester, UK \\ ${ }^{3}$ Anaesthetic Directorate, Aneurin Bevan University Health Board, Royal Gwent Hospital, Cardiff Road, Newport, Gwent, UK
}

\begin{abstract}
Aim of the study: To determine the characteristics, including the use of various diagnostic criteria, outcomes and treatment strategies in septic patients treated outside of the critical care area with pre-existing liver disease (LD).

Material and methods: The study population included patients recruited into two annual 24-hour prospective point-prevalence studies on the general wards and emergency departments across all Welsh acute hospitals in 2016 and 2017. Data were collected on patient demographics, observations and SIRS, SOFA and qSOFA scores.

Results: Out of 839 recruited patients, 24 (2.9\%) had a past medical history of LD. 12/24 (50\%) had a SIRS score $\geqslant 2,21 / 24(87.5 \%)$ a SOFA score $\geqslant 2$ and $3 / 24(12.5 \%)$ a qSOFA score $\geqslant 2$. Patients with LD had 2.4 times higher odds $(95 \% \mathrm{Cl}=1.07-5.53, p=0.03)$ of mortality after the sepsis episode. LD patients were younger than non-LD patients $(p=0.04$ ) but not significantly different in frailty, do not attempt cardiopulmonary resuscitation (DNA-CPR) status or ceiling of care ( $p=0.78, p=0.54, p=0.06$, respectively).

Conclusions: The 90-day mortality was greater in patients with LD than the rest of the population. Management of sepsis in LD patients poses a challenge with current therapeutic bundles being underused and of unclear significance in improving patient outcome.
\end{abstract}

Key words: sepsis, liver, screening.

\section{Address for correspondence}

Prof. Tamas Szakmany, Department of Anaesthesia, Intensive Care and Pain Medicine, Division of Population Medicine, Cardiff University, Heath Park Campus, Cardiff, UK, e-mail: szakmanyt1@cardiff.ac.uk

\section{Introduction}

Sepsis is defined as a dysregulated host response to infection resulting in acute organ dysfunction [1]. Despite the progress made in the clinical management of sepsis, sepsis morbidity and mortality rates remain high. Due to the various functions of the liver in sepsis, liver injury before or after the onset of infection has a crucial effect on the severity and outcome of sepsis in patients [2]. A patient with liver disease (LD) represents an immunocompromised host [3, 4], and therefore, patients with liver failure or chronic liver conditions are not only at higher risk of developing sepsis, but also suffer from increased morbidity and mortality after the sepsis episode [5]. Clinicians should therefore have a high index of suspicion for infection when cirrhotic patients are unwell or present with non-specific symptoms.

The aim of this study was to determine the characteristics, including the use of various diagnostic criteria, outcomes and treatment strategies in septic patients treated outside of the critical care area with pre-existing liver disease.

\section{Material and methods}

\section{Study design and participants}

Secondary analysis of outcomes for patients with liver disease was performed on a patient population recruited into two annual 24-hour prospective point-prev- 
alence studies on the general wards and emergency departments (ED) across all Welsh acute hospitals in 2016 and 2017. Patient data were collected using an innovative platform developed by the Welsh Digital Data Collection Platform, described in detail in our previous studies [6-8]. In brief, this multi-centre, prospective, observational study of patients with suspected sepsis was conducted in 14 hospitals in Wales with 24/7 consultant-level ED supervision and the facility to admit and treat any acutely unwell patient. We screened patients in the $\mathrm{ED}$ and the acute in-patient ward setting with suspected or proven infection on $19^{\text {th }}$ October 2016 and on the $18^{\text {th }}$ October 2017, both Wednesdays (08:00 to 07:59 hours the following day). These dates represented a typically "average" day in the National Health Service (NHS). LD was defined by the treating clinical teams, based on patients' history, elevated liver enzymes and cross-sectional imaging. In the LD group, all patients had acute or chronic liver problems documented by the medical team. Due to the methodology of the primary study, we did not attempt to further classify this group.

We included patients who fulfilled the following inclusion criteria: patient age of 18 or above, clinical suspicion of infection as documented in the medical notes and National Early Warning Score (NEWS) 3 or above. NEWS is a system for scoring the physiological measurements that are routinely recorded at the patient's bedside and includes respiration rate, oxygen saturation, systolic blood pressure, pulse rate, level of consciousness or new-onset confusion and temperature [9]. We excluded patients if they were less than 18 years of age or if they were already on intensive care or high dependency units. To facilitate linkage to national databases for the collection of follow-up data, we collected patient identifiable data using the secure data collection tool. We collected data on patient basic demographics, vital sign observations and Systemic Inflammatory Response Syndrome (SIRS) [10], Sequential Organ Failure Assessment (SOFA) [11] and qSOFA (quick SOFA) [12] scores. We also collected microbiological, radiological and laboratory data to facilitate analysis of sepsis source and its complications. Patients were followed up for 90 days for the survival analysis.
We aimed to recruit all eligible patients during the two point-prevalence studies and did not perform any formal sample size calculation.

The project was approved by the South Wales Regional Ethics Committee (16/WA/0071) and patients or legal representatives gave written informed consent. The Defining Sepsis on the Wards project was prospectively registered with an international trial registry (ISRCTN86502304).

\section{Statistical analysis}

Categorical variables are described as proportions and are compared using the chi square test. Continuous variables are described as median and range and compared using the Mann-Whitney $U$ test. A twotailed $p$-value $<0.05$ was considered statistically significant. All statistical tests were calculated using SPSS 25.0 (IBM Corp., Armonk, NY).

\section{Results}

\section{Patient characteristics}

In our study we screened 12,477 patients over the two 24-hour study periods in 14 Welsh hospitals. 839 patients had NEWS $\geq 3$ and documented clinical suspicion of infection and were recruited to the study. Out of all recruited patients, 24 (2.9\%) had a past medical history of liver disease. Baseline characteristics are summarised in Table 1.

Out of these 24 patients, 4 had biopsy or image proven cirrhosis, 3 had other signs of LD and 1 had signs of mild hepatic encephalopathy. Median Model for End-Stage Liver Disease (MELD) score in the LD cohort was 13.5 (IQR 7-19). The most common comorbidities in the LD group were chronic obstructive pulmonary disease (COPD) and hypertension.

The LD group appeared to have more comorbidities. To clarify this we calculated the comorbidities variables again and compared the number of comorbidities of the LD group with all the comorbidities apart from liver disease. In this setting, the median

Table 1. Patient characteristics. Values are number (proportion) or median (range). Comparison between liver disease and non-liver disease cohort was performed using the chi square or Mann-Whitney $U$ test. $P$ values of less than 0.05 are bold

\begin{tabular}{lcccc}
\hline Patient characteristic & All patients $(\boldsymbol{N}=\mathbf{8 3 9})$ & Liver disease $(\boldsymbol{n}=\mathbf{2 4})$ & Non-liver disease $(\boldsymbol{n}=\mathbf{8 1 5})$ & $P$ value \\
\hline Age, median (range) & $73(18-103)$ & $62.5(25-89)$ & $73(18-103)$ & 0.04 \\
\hline Male sex, $n(\%)$ & $411(49)$ & $16(66.7)$ & $385(47.2)$ & 0.09 \\
\hline Smoker, $n(\%)$ & $111(13.2)$ & $4(16.7)$ & $107(13.1)$ & 0.67 \\
\hline Number of comorbidities, median (range) & $1(0-5)$ & $2(1-4)$ & $1(0-5)$ & $<0.001$ \\
\hline
\end{tabular}




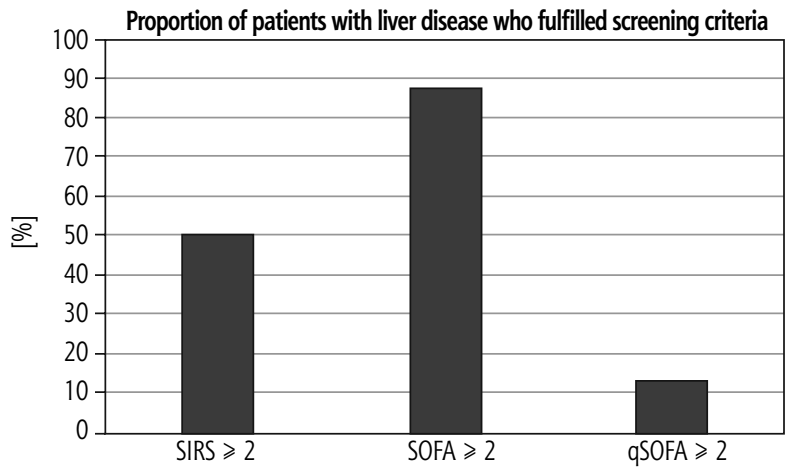

Fig. 1. Sepsis criteria in liver disease patient cohort

Table 2. Comparison of sepsis screening tool scores for liver disease and nonliver disease patients. Comparison between sepsis screening tool scores was performed using Mann-Whitney $U$ test. $P$ value of less than 0.05 is bold

\begin{tabular}{lccc}
\hline Sepsis screening tool & $\begin{array}{c}\text { Liver disease } \\
(\boldsymbol{n}=\mathbf{2 4})\end{array}$ & $\begin{array}{c}\text { Non-liver disease } \\
(\boldsymbol{n}=\mathbf{8 1 5})\end{array}$ & $\boldsymbol{P}$ value \\
\hline NEWS, median (range) & $4(3-11)$ & $4(3-16)$ & 0.47 \\
\hline SIRS, median (range) & $2(1-4)$ & $2(0-4)$ & 0.08 \\
\hline SOFA, median (range) & $4(0-11)$ & $2(0-14)$ & $\mathbf{0 . 0 0 1}$ \\
\hline qSOFA, median (range) & $1(0-2)$ & $1(0-3)$ & 0.73 \\
\hline
\end{tabular}

Table 3. Impact of comorbidities on patient survival. Data on comorbidities was missing for 36 out of 849 patients. The comparison was performed using chi-square. COPD - chronic obstructive pulmonary disease, IHD - ischaemic heart disease. $P$ value of less than 0.05 is bold

\begin{tabular}{lccc}
\hline Comorbidity & $\begin{array}{c}\text { Survivors } \\
(\boldsymbol{n}=\mathbf{5 9 9 )}\end{array}$ & $\begin{array}{c}\text { Non-survivors } \\
(\boldsymbol{n}=\mathbf{2 1 4})\end{array}$ & $\boldsymbol{P}$ value \\
\hline Liver disease, $n(\%)$ & $13(2.17)$ & $11(5.14)$ & 0.03 \\
\hline COPD, $n(\%)$ & $180(30.05)$ & $50(23.36)$ & 0.06 \\
\hline Diabetes, $n(\%)$ & $109(18.19)$ & $34(15.88)$ & 0.49 \\
\hline Hypertension, $n(\%)$ & $196(32.72)$ & $76(35.51)$ & 0.46 \\
\hline IHD, $n(\%)$ & $99(16.53)$ & $46(21.49)$ & 0.11 \\
\hline Neuromuscular, $n(\%)$ & $18(2.51)$ & $11(5.14)$ & 0.15 \\
\hline
\end{tabular}

Table 4. Comparison of physiological reserve factors in patients with LD in comparison to non-LD patients. Data on selected variables were missing for 38 out of 849 patients Values are number (proportion) or median (range). Comparison between liver disease and non-liver disease cohort was performed using the chi-square or Mann-Whitney $U$ test

\begin{tabular}{lccc}
\hline Variable & $\begin{array}{c}\text { Liver disease } \\
(\boldsymbol{n}=\mathbf{2 4})\end{array}$ & $\begin{array}{c}\text { Non-liver disease } \\
(\boldsymbol{n}=\mathbf{7 7 7 )}\end{array}$ & $\boldsymbol{P}$ value \\
\hline $\begin{array}{l}\text { Clinical frailty score, } \\
\text { median (range) }\end{array}$ & $4(2-9)$ & $5(1-9)$ & 0.78 \\
\hline DNA-CPR, $n(\%)$ & $2(8.7)$ & $202(26.0)$ & 0.06 \\
\hline Ceiling of care, $n(\%)$ & $4(17.4)$ & $177(22.8)$ & 0.54 \\
\hline
\end{tabular}

DNA-CPR - do not attempt cardiopulmonary resuscitation

for both groups was 1 (range: 0-3 for LD patients and $0-5$ for non-LD patients, $p=0.6$ ).

\section{Sepsis screening tool analysis}

Out of all recruited patients with a history of liver disease, 12/24 (50\%) had a SIRS score $\geq 2,21 / 24$ (87.5\%) had a SOFA score $\geq 2$ and 3/24 (12.5\%) had a qSOFA score $\geq 2$ (Fig. 1). Significantly more LD patients than non-LD patients had SOFA $\geq 2(p=0.009)$. Moreover, LD patients had higher total SOFA scores than non-LD patients (Table 2).

All patients with LD who had an acute (over a period of $24 \mathrm{~h}$ ) increase in the SOFA $\geq 2$ scored in the respiratory, cardiovascular or renal subscores alone, whilst some patients scored on their bilirubin and platelet count values, which could represent the underlying LD.

The difference in median scores for NEWS, SIRS and qSOFA between LD and non-LD patients were not statistically significant (Table 2). Out of all LD patients $15 / 24(62.5 \%)$ had chest infection, $3 / 24$ (12.5\%) urinary tract infection and 11/24 (25\%) intra-abdominal infection.

\section{Survival analysis}

Out of 839 recruited patients, 222 (26.6\%) were non-survivors. The analysis of liver disease cohort revealed that $11 / 24(45.8 \%)$ of LD patients were nonsurvivors in comparison to $211 / 815$ (25.9\%) non-LD patients. Patients with pre-existing liver disease had 2.4 times higher odds (95\% CI $=1.07-5.53, p=0.03$ ) of mortality after the sepsis episode. None of the other comorbidities had a significant impact on the survival (Table 3). There was a higher MELD score in non-survivor $\mathrm{LD}$ patients in comparison to $\mathrm{LD}$ patients who survived, but it was not significant; median 16 vs. $8, p=0.07$.

\section{Physiological reserve and sepsis management}

We also performed analysis of variables associated with patient characteristics and treatment in hospital to identify differences in patient physiological reserve and sepsis management (Table 4). LD patients were not significantly different than non-LD patients in terms of their frailty $(p=0.78)$. Both patient cohorts were also not statistically different in regards to do not attempt cardiopulmonary resuscitation (DNA-CPR) order in place $(p=0.06)$ and ceiling of care $(p=0.54)$.

Only 15/24 (62.5\%) of septic LD patients received intravenous antibiotics and 8/24 (33.3\%) had blood cultures taken. The Sepsis Six pathway was fulfilled for $2 / 24(8.3 \%)$ and $3 / 24(12.5 \%)$ patients were seen by a senior clinician. Interestingly, the management was not significantly different from the septic non-LD cohort ( $p=0.98, p=0.79, p=0.52, p=0.75$ respectively) 
(Table 5). The most commonly used antibiotic in both cohorts was piperacillin with tazobactam (29.2\% of LD patients and $24.8 \%$ of non-LD patients).

\section{Discussion}

Infection is responsible for over $50 \%$ of admissions of cirrhotic patients to hospitals and is one of the main precipitants for the development of multiple organ failure and death [13]. Our study shows that patients with pre-existing liver disease are at increased risk of mortality after the sepsis episode, despite having similar clinical observations, physiological reserve, received care and being younger than the non-liver disease cohort.

Our data on patient survival are similar to other studies which recruited patients with sepsis outside of the critical care units [14-16]. Liver disease is associated with immune deficiency, which leads to a reduced ability for the organism to prevent or clear infectious agents [17]. It has been suggested that there exists a crucial 'golden window' period, during which reversing the acute infective insult and preventing development of organ dysfunction could support hepatic regeneration and facilitate spontaneous recovery [18]. Studies have shown that early diagnosis of sepsis and quick implementation of therapeutic bundles facilitate reduction of the incidence of severe complications and decrease patient morbidity and mortality $[19,20]$. The data from our cohort suggest that when multi-organ dysfunction has already developed, such as in most of the patients in the LD cohort, outcomes are particularly poor. It appears that SOFA-based criteria identify most of the patients with liver disease at risk of sepsis, an observation in line with our previous data for all sepsis patients [21, 22]. Whilst SOFA scores can be influenced by chronic stigmata of LD such as low platelet count and high bilirubin, in our cohort patients with LD had an acute (over a period of $24 \mathrm{~h}$ ) increase of the SOFA score by 2 or more in most cases due to respiratory problems and in some instances due to cardiovascular instability.

Our study also highlights the low rates of antibiotics use, obtaining blood cultures and delivery of the Sepsis Six bundle in the patients with LD who are at increased risk of death from infection [23]. In line with previous reports, the mortality of patients with liver disease is significantly higher than the non-LD cohort despite similar hospital management $[24,25]$. This could indicate that patient outcomes are primarily determined by their underlying chronic conditions and that the modifiable effect of treating a potential infection where an organ dysfunction is already established might not significantly change patient outcomes [26]. Appropriate management of the underlying condition,
Table 5. Comparison of management of sepsis patients with $L D$ in comparison to non-LD patients. Data on selected variables was missing for 26 out of 849 patients. Comparison between liver disease and non-liver disease cohort was performed using the chi-square test

\begin{tabular}{lccc}
\hline Management & $\begin{array}{c}\text { Liver-disease } \\
(\boldsymbol{n}=\mathbf{2 4 )}\end{array}$ & $\begin{array}{c}\text { Non-liver disease } \\
(\boldsymbol{n}=\mathbf{7 8 9})\end{array}$ & $\boldsymbol{P}$ value \\
\hline IV antibiotics, $n(\%)$ & $15(62.5)$ & $493(60.5)$ & 0.98 \\
\hline Blood culture, $n(\%)$ & $8(33.3)$ & $217(40.3)$ & 0.79 \\
\hline Sepsis Six pathway, $n(\%)$ & $2(8.3)$ & $100(12.3)$ & 0.52 \\
\hline $\begin{array}{l}\text { Seen by senior clinician, } \\
n(\%)\end{array}$ & $3(12.5)$ & $85(10.4)$ & 0.75 \\
\hline
\end{tabular}

rapid identification of infection and prevention of the development of further organ dysfunction could have the biggest impact on patient survival. However, given the less than adequate delivery of basic sepsis care, it could also be argued that this inappropriate management had a significant effect on the outcomes. As other studies reported similarly poor adherence to the early sepsis bundle elements, it is impossible to establish a causative mechanism from the current data [24].

Further research is required to explore immunological mechanisms that lead to the increased predisposition to infection and worse outcomes in patients with pre-existing liver disease [27]. This could enable development of targeted immunotherapeutic strategies to improve patient survival in well-defined subgroups [28].

The strengths of our study include wide participation of centres including University Hospitals and District General Hospitals and prospectively collected patient information. Our study has high internal validity, as our previous four studies applied the same methodology and recruited a similar number of patients in the same centres $[21,22,29]$. We also obtained a comprehensive dataset and substantial follow-up time [23].

Our study has some significant limitations. The number of patients with liver disease in our cohort is small. However, the proportion of patients with LD is similar to data described in other epidemiological studies [30,31]. Due to the small sample size, our results should be considered as exploratory, and would need to be confirmed in larger, prospective studies. We were also not able to obtain information about the aetiology of liver disease or establish the cause of death based on the official death certificate. The most common types of liver disease in the UK are alcohol, obesity and viral illness related, and it has been observed that alcohol related admissions are frequent in the Welsh Intensive Care Units [32, 33]. Nevertheless, due to the small size of the cohort, it is unlikely that 
these data would be beneficial in the analysis of patient mortality [7].

\section{Conclusions}

The 90-day mortality was greater in patients with pre-existing liver disease than the rest of the population. It appears that SOFA-based criteria identify most of the patients with liver disease at risk of sepsis, an observation in line with our previous data for all sepsis, despite elements of the SOFA score implicating chronic liver disease. The management of sepsis in patients with liver disease still poses a challenge, with current therapeutic bundles being underused and of unclear significance in improving patient outcomes. It appears that patient survival could be primarily determined by the patient's underlying condition and could be improved by treatment of the condition and prevention of the infection.

\section{Funding}

This research was funded by Fiona Elizabeth Agnew Trust: Features Award 2016 and Welsh Intensive Care Society Research Award 2015. The APC was funded by Fiona Elizabeth Agnew Trust: Features Award 2016.

\section{Acknowledgments}

\section{Welsh Digital Data Collection Platform Collaborators:}

Ben Sharif, Sian Cleaver, Naomi Spencer, Amit Kurani, Camilla Lee, Jessica Davis, Carys Durie, Jude Joseph-Gubral, Angelica Sharma, Lucy Allen, Billie Atkins, Alex Gordon, Llewelyn Jones, Amy Noble, Matthew Bradley, Henry Atkinson, Joy Inns, Harriet Penney, Carys Gilbert, Rebecca Walford, Louise Pike, Ross Edwards, Robyn Howcroft, Hazel Preston, Jennifer Gee, Nicholas Doyle, Charlotte Maden, Claire Smith, Nik Syakirah Nik Azis, Navrhinaa Vadivale, Richard Pugh, Ceri Battle, Ronan Lyons, Paul Morgan, Peter Havalda, Wojciech Groblewski, Andrew Campbell, Maria Hobrok, Igor Otahal, Vincent Hamlyn, Luis Macchiavello, Rhidian Jones, Orsolya Minik, Paul Morgan, Gemma Ellis, Ceri Brown, Chris Littler, Chris Subbe, Judith E. Hall, Szilvia Szoke, Richard Self, Robert Lundin, Meshari Alsaeed, Hannah Williams, Arfa Ayob, Nor Farzana, Sweta Parida, David Lawson, Michal Mazur, Lezia D'Souza, Bethan Ponting, Terrance Lau, Ruairidh Kerrigan, Lucy Morgan, Roshan Vindla, Claudia Zeicu, Becky James, Amirah Amin Ariff, Wan Binti Wan Az- zlan, Charlotte Collins, Elizabeth Wickens, Alisa Norbee, Aliya Zulkefli, Thomas Haddock, Megan Thomas, Matthew Lee, Akshita Dandawate, Holleh Shayan-Arani, Ellie Taylor, Oliver Kyriakides, Rachel Price, Ffion Haf Mackey, Emily Haines, Samuel Chun, Chantal Roberts, Alessia Waller, Laura Heekin, Kathy Wang, Rhianna Church, Shrina Patel, Marianne Broderick, Hannah Whillis, Daniel Craig Hathaway, Emel Yildirim, Caitlin Atkins, Adam George Mounce, Anoopama Ramjeeawon, Ndaba Mtunzi, Duncan Soppitt, Jay Hale, Jack Wellington, Robert Buchanan Ross, Danielle Lis, Sophie Stovold, Sam Vickery, Nia Jones, Alice O’Donnell, Monty Cuthbert, Osa Eghosa, Muhammad Karim, Lowri Williams, Louise Tucker, Thomas Downs, Ailsa MacNaught, Swagath Balachandran, Abbie Shipley, Jennifer Louise Kent, Talea Roberts, Samuel Tilley, Bethany Davies, Emma Withers, Krishna Parmar, Lucie Webber, Thomas Grother, Harry Smith, Rachel Watson, Natalie Hoyle, Rym Chafai El Alaoui, Omar Marei, Emma Kirby, Anna Gilfedder, Lydia Maw, Sarah O’Connor, Abigail Rogers, Zhao Xuan Tan, Clare Chantrill, Amal Robertson, Jonathan Foulkes, Rahana Khanam, Jomcy John, Isobel Sutherland, Sarah Hannah Meehan, Huria Metezai, Hannah Dawson, Eloise Baxendale, Karishma Khan, Pan Myat, Andrew Forrester, Oliver Moore, Hse Juinn Lim, Aimee Owen, Faris Hussain, Nima-banu Allybocus, Maneha Sethi, Umair Asim, Emelia Boggon, Ibrahim Alkurd, Genevieve Lawrence, Jade Brown, Lowri Hughes Thomas, Emily Murphy, Evie Lambert, Jeremy Guilford, Beth Payne, Mariam Almulaifi, Arwel Poacher, Sashiananthan Ganesananthan, Sara Tanatova, Jasmine Kew, Megan Eilis Clark, Ellen Hannay, Olesya Godsafe, Christina Houghton, Francesca Lavric, Rachel Mallinson, Hei Man Priscilla Chan, Eshen Ang, Niamh McSwiney, Yin Yin Lim, Zong Xuan Lee, Svetlana Kulikouskaya, Nur Zulkifli, Sheryl Lim, Lim Xin, Thomas Chandy, Abduahad Taufik, James Cochrane, Sioned Davies, Samuel Willis, John Lynch, Sieh Yen Heng, Alex Cooper, Henrik Graf von der Pahlen, Isabella Talbot, Robin Gwyn Roberts, Jessica Sharma Smith, Aisling Sweeney, Cerian Roberts, Paul McNulty, Elin Walters, Robert Sinnerton, Benjamin Tanner, Berenice Cunningham-Walker, Chloe Spooner, Akanksha Kiran, Nabeegh Nadeem, Vidhi Unadkat, John Ng Cho Hui, Esme Sparey, David Li, Jessica Smith, India Corrin, Harry Waring, Adeel Khan, Emily Baker, Mohammad Yahya Amjad, Miriam Cynan, Imogen Hay, Catherine Russell, Joseph Davies, Rebecca Parsonson, Ajitha Arunthavarajah, Jessica Nicholas, Aaron Harris, Tim Burnett, Josephine Raffan Gowar, Sam DeFriend, Helena Jones, Nur Amirah Binti Maliki, Mark Zimmerman, Jessica Webber, Rebecca Phillips, Lauren McCarthy, Lara Wirt, Emily Hubbard, Emily Evans, Laura Jane Davis, Llywela 
Wyn Davies, Lee Sanders-Crook, Amrit Dhadda, Genna Logue, Isabel Jones, Adiya Urazbayeva, Nur Haslina Ahmad Hanif, Yau Ke Ying, Alice Coleclough, Eilis Higgins, Tze Gee Ng, Sam Booth, Nilarnti Vignarajah, Tessa Chamberlain, Dongying Zhao, Nayanatara Nadeesha Tantirige, John Watts, Amy Prideaux, Amelia Tee, Annabelle Hook, Adam Mounce, Emily Eccles, Kirtika Ramesh, Laura Bausor, Amy Handley, Rebecca Paddock, Lopa Banerjee.

\section{Disclosure}

The authors declare no conflict of interest.

\section{References}

1. Singer M, Deutschman CS, Seymour CW, et al. The third international consensus definitions for sepsis and septic shock (Sepsis-3). JAMA 2016; 315: 801-810.

2. Yan J, Li S, Li S. The role of the liver in sepsis. Int Rev Immunol 2014; 33: 498-510.

3. Canabal JM, Kramer DJ. Management of sepsis in patients with liver failure. Curr Opin Crit Care 2008; 14: 189-197.

4. Hotchkiss RS, Monneret G, Payen D. Immunosuppression in sepsis: a novel understanding of the disorder and a new therapeutic approach. Lancet Infect Dis 2013; 13: 260-268.

5. Gustot T, Felleiter P, Pickkers P, et al. Impact of infection on the prognosis of critically ill cirrhotic patients: results from a large worldwide study. Liver Int 2014; 34: 1496-1503.

6. Sharif B, Lundin RM, Morgan P, et al. Developing a digital data collection platform to measure the prevalence of sepsis in Wales. J Am Med Inform Assoc 2016; 23: 1185-1189.

7. Kopczynska M, Sharif B, Pugh R, et al. Prevalence and outcomes of acute hypoxaemic respiratory failure in Wales: The PANDORAWALES Study. J Clin Med 2020; 9: 3521.

8. Frankling C, Patel J, Sharif B, et al. A snapshot of compliance with the sepsis six care bundle in two acute hospitals in the West Midlands, UK. Indian J Crit Care Med 2019; 23: 310-315.

9. Smith GB, Prytherch DR, Meredith P, et al. The ability of the National Early Warning Score (NEWS) to discriminate patients at risk of early cardiac arrest, unanticipated intensive care unit admission, and death. Resuscitation 2013; 84: 465-470.

10. Bone RC, Balk RA, Cerra FB, et al. Definitions for sepsis and organ failure and guidelines for the use of innovative therapies in sepsis. Chest 1992; 101: 1644-1655.

11. Vincent JL, Moreno R, Takala J, et al. The SOFA (Sepsis-related Organ Failure Assessment) score to describe organ dysfunction/failure. On behalf of the Working Group on Sepsis-Related Problems of the European Society of Intensive Care Medicine. Intensive Care Med 1996; 22: 707-710.

12. Raith EP, Udy AA, Bailey M, et al. Prognostic Accuracy of the SOFA Score, SIRS Criteria, and qSOFA score for in-hospital mortality among adults with suspected infection admitted to the intensive care unit. JAMA 2017; 317: 290-300.

13. Bajaj JS, O'Leary JG, Reddy KR, et al. Second infections independently increase mortality in hospitalized patients with cirrhosis: the North American consortium for the study of endstage liver disease (NACSELD) experience. Hepatology 2012; 56: $2328-2335$

14. Rhodes A, Evans LE, Alhazzani W, et al. Surviving sepsis campaign: international guidelines for management of sepsis and septic shock: 2016. Intensive Care Med 2017; 43: 304-377.
15. Finkelsztein EJ, Jones DS, Ma KC, et al. Comparison of qSOFA and SIRS for predicting adverse outcomes of patients with suspicion of sepsis outside the intensive care unit. Crit Care 2017; 21: 73.

16. Sakr Y, Jaschinski U, Wittebole X, et al. Sepsis in intensive care unit patients: worldwide data from the intensive care over nations audit. Open Forum Infect Dis 2018; 5: 313.

17. Albillos A, Lario M, Álvarez-Mon M. Cirrhosis-associated immune dysfunction: Distinctive features and clinical relevance. J Hepatol 2014; 61: 1385-1396.

18. Sarin SK, Choudhury A. Acute-on-chronic liver failure: terminology, mechanisms and management. Nat Rev Gastroenterol Hepatol 2016; 13: 131-149.

19. Woźnica E, Inglot M, Woźnica R, Łysenko L. Liver dysfunction in sepsis. Adv Clin Exp Med 2018; 27: 547-551.

20. Burke J, Wood S, Hermon A, Szakmany T. Improving outcome of sepsis on the ward: introducing the 'Sepsis Six' bundle. Nurs Crit Care 2019; 24: 33-39.

21. Szakmany T, Pugh R, Kopczynska M, et al. Defining sepsis on the wards: results of a multi-centre point-prevalence study comparing two sepsis definitions. Anaesthesia 2017; 73: 195-204.

22. Kopczynska M, Sharif B, Cleaver S, et al. Red-flag sepsis and SOFA identifies different patient population at risk of sepsis-related deaths on the general ward. Medicine 2018; 97: e13238.

23. Kopczynska M, Sharif B, Unwin H, et al. Real world patterns of antimicrobial use and microbiology investigations in patients with sepsis outside the critical care unit: secondary analysis of three nation-wide point prevalence studies. J Clin Med 2019; 8: 1337.

24. Cheng B, Xie G, Yao S, et al. Epidemiology of severe sepsis in critically ill surgical patients in ten university hospitals in China. Crit Care Med 2007; 35: 2538-2546.

25. Angus DC, Linde-Zwirble WT, Lidicker J, et al. Epidemiology of severe sepsis in the United States: Analysis of incidence, outcome, and associated costs of care. Crit Care Med 2001; 29: 1303-1310.

26. Rhee C, Jones TM, Hamad Y, et al. Prevalence, underlying causes, and preventability of sepsis-associated mortality in US acute care hospitals. JAMA Netw Open 2019; 2: e187571.

27. Rubio I, Osuchowski MF, Shankar-Hari M, et al. Current gaps in sepsis immunology: new opportunities for translational research. Lancet Infect Dis 2019; 19: e422-e436.

28. Selvapatt N, Singanayagam A, Wendon J, Antoniades CG. Understanding infection susceptibility in patients with acute-onchronic liver failure. Intensive Care Med 2014; 40: 1363-1366.

29. Szakmany T, Lundin RM, Sharif B, et al. Sepsis prevalence and outcome on the general wards and emergency departments in wales: results of a multi-centre, observational, point prevalence study. PLoS One 2016; 11: e0167230.

30. Byass P. The global burden of liver disease: a challenge for methods and for public health. BMC Med 2014; 12: 159.

31. Mokdad AA, Lopez AD, Shahraz S, et al. Liver cirrhosis mortality in 187 countries between 1980 and 2010: a systematic analysis. BMC Med 2014; 12: 145.

32. Williams R, Alexander G, Aspinall R, et al. Gathering momentum for the way ahead: fifth report of the Lancet Standing Commission on Liver Disease in the UK. Lancet 2018; 392: 2398-2412.

33. Lynch C, Pugh R, Battle C, Welsh Intensive Care Society Audit and Research Group. A multicentre prospective evaluation of alcohol-related admissions to intensive care units in Wales. J Intensive Care Soc 2017; 18: 193-197. 